\title{
Avaliação da Função Renal e Alterações Morfológicas em Ratos Tratados com Dipirona em Diferentes Doses
}

\section{Evaluation of Renal Function and Morphological Changes in rats Treated with Dipyrone in Different Dosing}

\author{
Bárbara Soares Correa ${ }^{\text {**; }}$ Carolina Aparecida Campaninia; Laís Cristina Monteiro Paiva; \\ Ramiro Nunes da Silva ${ }^{a}$; Wilson Roberto Malfaráa; Ana Rosa Criscia \\ ${ }^{a}$ Centro Universitário Barão de Mauá. Ribeirão Preto/SP. \\ *E-mail: barbaraasoares@hotmail.com \\ Recebido em: 09/09/2015; Aceito em: 21/10/2015
}

\begin{abstract}
Resumo
A dipirona é um fármaco muito utilizado para o tratamento da febre e da dor, entretanto, seus efeitos em doses altas podem levar a danos irreversíveis e até letais. $\mathrm{Na}$ presente investigação foram utilizados 32 ratos albinos machos da linhagem Wistar. Um grupo (1) recebeu uma dose única, $5.000 \mathrm{mg} / \mathrm{Kg}$ de dipirona por gavagem e o outro grupo (2) administração uma vez ao dia, por quatro dias consecutivos, de $600 \mathrm{mg} / \mathrm{Kg}$ de dipirona. Ao final de quatro dias, para o grupo 2 e após 3 horas de ingestão do medicamento para o grupo 1 , os animais foram eutanasiados em câmara de $\mathrm{CO} 2$, conforme a aprovação do Comitê de Ética Animal do Centro Universitário Barão de Mauá (CEPAn). Foram avaliados os parâmetros ureia e creatinina e a histopatologia renal. Os resultados dos escores hemorragia glomerular, congestão renal e processo inflamatório foram estatisticamente significativos nos três parâmetros analisados para dose única; congestão renal e processo inflamatório para dose múltipla. O marcador de função renal, ureia apresentou diferença estatística significativa com a dose única. Os resultados do modelo experimental atestam que a metodologia aplicada foi capaz de demonstrar as alterações histopatológicas do grupo tratado com dipirona dose única assim como alterações na dosagem de ureia e a preservação das características normais do grupo controle. O mesmo não ocorreu com o grupo tratado com doses múltiplas.
\end{abstract}

Palavras-chave: Dipirona. Rim. Ratos Wistar.

\begin{abstract}
Dipyrone is a drug widely used for the treatment of fever and pain, but their effects at high doses can lead to irreversible and even lethal damages.In the present study it was used 32 male albino rats of Wistar strain. First group received a single dose $5.000 \mathrm{mg} / \mathrm{kg}$ by gavage dipyrone and the second given once daily for four consecutive days of $600 \mathrm{mg} / \mathrm{kg}$ Dipyrone. At the end of four days, for group 2 and 3 hours after ingestion of the drug for group 1, the animals were euthanized in a CO2 chamber, as the approval of the Baron of Maua University Center (Cepan) Animal Ethics Committee. The urea and creatinine parameters and renal histopathology were evaluated. The results of the scores glomerular bleeding, renal congestion and inflammation were statistically significant in all parameters analyzed for single dose; renal congestion and inflammation to multiple dose. The marker of renal function, urea statistically significant difference with the single dose. The results of the experimental model show that the methodology was able to demonstrate the histopathological changes in the group treated with single dose dipyrone as well as changes in the levels of urea and the preservation of the normal characteristics of the control group. This did not occur with the group treated with multiple doses.
\end{abstract}

Keywords: Dipyrone. Kidney.Wistar Rats

\section{Introdução}

A dipirona é um analgésico comum usado em todo o mundo por adultos, crianças e animais ${ }^{1}$, sendo consumido em diversas apresentações farmacêuticas (solução oral, injetável, comprimidos e supositórios), e comercializado principalmente como medicamento isento de prescrição médica ${ }^{2}$.

Sendo assim, é um dos medicamentos mais utilizados inclusive no meio universitário, como a formulação medicamentosa mais comum em estudos sobre automedicação ${ }^{3}$.

Desde 1922 quando foi introduzida no Brasil com o nome de novalgina, sua comercialização tornou-se mundialmente crescente até a década de 1970, quando foram relatados casos de anemia aplástica e principalmente de agranulocitose, que é caracterizada pela ausência total de neutrófilos circulantes, condição clínica de déficit imunológico potencialmente fatal, exigindo a suspensão do tratamento ${ }^{4}$.
Entretanto, na Alemanha, há alguns anos, já havia sido retirado do mercado todas as associações contendo dipirona, pois, além de seus efeitos sobre a medula óssea, a dipirona também pode causar reações cutâneas, tais como reações alérgicas idiossincráticas, broncoespasmo, choque anafilático, necrose epidérmica tóxica, hepatite e hipotensão severa 5 .

A dipirona faz parte da classe dos anti-inflamatórios não esteroide (AINES), derivado pirazolônico, e possui quatro ações principais: analgésico, antitérmico, anti-inflamatórios e espasmolítico. Possui também excelente ação antiartrítica, todas as quais decorrem principalmente da inibição da enzima cicloxigenase e consequente redução na síntese de prostaglandina e tromboxanos; este fato é responsável pelas muitas respostas adversas ao medicamento, ou seja, ulcerações gastrintestinais, nefro e hepatopatias, retenção hídrica, eventos trombóticos e comprometimento cardiovascular e outros ${ }^{6}$.

São as prostaglandinas produzidas pelos rins, as 
substâncias responsáveis por manter o fluxo sanguíneo renal e a taxa de filtração glomerular, promovendo a vasodilatação, diminuição da resistência vascular e melhora da perfusão renal, pela redistribuição do fluxo sanguíneo do córtex para a região justamedular. As prostaglandinas (PGD) promovem vasodilatação com a diminuição da liberação de norepinefrina, e antagoniza a ação vasoconstritora da angiotensina II nas arteríolas aferentes ${ }^{7}$.

A dipirona é uma das drogas com excreção renal. O sistema renal é o principal sistema de excreção das drogas, sendo o rim o órgão mais importante nesse sistema.

A excreção renal de uma droga do organismo depende da lipossolubilidade da droga, taxa de filtração glomerular, $\mathrm{pH}$ do filtrado, secreção tubular ativa e reabsorção tubular ativa. Altas concentrações de uma determinada droga na luz dos túbulos renais podem desencadear respostas inflamatórias e comprometer a função de filtração glomerular, principalmente em pacientes com morbidade e quando associado à outra droga, como diuréticos ou uso abusivo de analgésicos ${ }^{8}$.

Diante do exposto, o objetivo desta investigação foi analisar possíveis lesões renais, como, processo inflamatório, esclerose glomerular e congestão vascular, nas diferentes doses aplicadas e a função renal mediante dosagem das concentrações plasmáticas de ureia e creatinina.

\section{Material e Métodos}

Na presente investigação foram utilizados 32 ratos albinos machos da linhagem Wistar, cujos pesos variavam entre $200 \mathrm{~g}$ a $250 \mathrm{~g}$. Esses animais foram divididos em quatro grupos. O grupo 1 (dose única) composto por 16 animais, foi subdividido em grupo controle (GCU) com oito animais que receberam 1,0 ml/100 $\mathrm{g}$ de peso corporal de água destilada por gavagem em administração única; e o grupo tratado (GDipU) com oito animais que receberam $5.000 \mathrm{mg} / \mathrm{Kg}$ de dipirona, o que corresponde a $1.250 \mathrm{mg}$ de dipirona para cada animal de $250 \mathrm{~g}$ ou 50 gotas para o mesmo animal pesando $250 \mathrm{~g}$.

O grupo 2 (doses múltiplas), composto por 16 animais, foi subdividido em grupo controle (GCM) com oito animais que receberam 1,0 ml/100 g peso corporal de água destilada por gavagem em administração uma vez ao dia por quatro dias consecutivos; e o grupo tratado (GDipM) com oito animais que receberam $600 \mathrm{mg} / \mathrm{Kg}$ de dipirona, o que corresponde a 150 mg de dipirona para cada animal de $250 \mathrm{~g}$ ou a 6 gotas para o mesmo animal pesando $250 \mathrm{~g}$, administrados uma vez ao dia, durante quatro dias consecutivos. Ao final de quatro dias, para o grupo 2 e após 3 horas de ingestão do medicamento para o grupo 1, os animais foram eutanasiados, em câmara de CO2, conforme a aprovação do Comitê de Ética Animal do Centro Universitário Barão de Mauá (CEPAn), processo nº 209/2014.

$O$ sangue foi coletado da veia cava inferior, aproximadamente $5 \mathrm{ml}$ para os exames laboratoriais e os rins direito e esquerdo, foram retirados para os procedimentos histológicos de rotina.
Para avaliar os possíveis efeitos tóxicos sobre a função renal após a administração das respectivas doses nos dois grupos experimentais, foram avaliados os parâmetros ureia e creatinina.

Para esse procedimento, as amostras de sangue dos animais foram coletadas em tubo gel separador de células para a dosagem de ureia $(\mathrm{mg} / \mathrm{dL})$ e creatinina $(\mathrm{mg} / \mathrm{dL})$. Após aproximadamente 30 minutos do procedimento de coleta, as amostras de sangue foram centrifugadas e o plasma obtido reservado para a análise. A determinação da concentração das substâncias foi realizada segundo protocolo experimental descrito na bula do kit, especificado pelo fabricante da DIALAB. A determinação espectofométrica foi realizada em aparelho automatizado BS-200, marca Mindray, do Ambulatório "Domingos João Batista Spinelli”, em Ribeirão Preto.

Na laparotomia realizada em cada animal, retirou-se o rim para análise histopatológica, ou seja, fixação em formol 10\%, inclusão em parafina e coloração com Hematoxilina e Eosina (H.E.).

Em seguida, as lâminas foram analisadas em microscópio de luz com objetivas planas, acromáticas com aumento de 100 a 400 vezes, coloradas pela hematoxilina e eosina.

Os rins foram analisados morfologicamente de forma comparativa entre os 2 grupos, experimental e controle de ambas as doses, múltiplas e única.

O sistema de escore foi utilizado para graduar as lesões presentes nos rins, baseado na presença e intensidade das alterações.

Os parâmetros analisados foram esclerose glomerular, processo inflamatório e congestão vascular com escores de zero a três para cada um deles, na dependência da intensidade das alterações, sendo considerado zero: histologia normal; 1, 2 e 3: comprometimento leve, moderado e severo, respectivamente ${ }^{9}$.

Em cada lâmina, foram analisados três campos de médio aumento (100X).

Para comparar as médias dos escores das lesões renais nas duas doses, realizou-se o Mann-Whitney Test.

\section{Resultados e Discussão}

Os estudos com animais destinados a elucidar os mecanismos de lesões renais com o uso de analgésicos têm sido dificultados devido a lesão ocorrer somente depois de doses muito elevadas e administradas por longos períodos; no entanto, ratos Wistar parecem ser mais sensíveis ${ }^{15}$, razão pela qual ter sido a espécie escolhida para esta investigação.

Neste estudo, avaliou-se a função renal de 32 ratos Wistar que receberam uma alta dose de dipirona com uma dosagem única e com doses múltiplas em quatro dias consecutivos, por meio da concentração plasmática de ureia e creatinina e da histopatologia do rim.

O Gráfico da Figura 1 representa os efeitos dos tratamentos 
com dose única e múltipla de dipirona via gavagem; no marcador de função renal, ureia, o resultado mostrou diferença estatística significativa ( $\mathrm{p}>0,006)$ em dose única. Entretanto, o mesmo não aconteceu com doses múltiplas $(\mathrm{p}>0,5)$.

Figura 1: Dosagem de ureia nos dois tipos de tratamentos

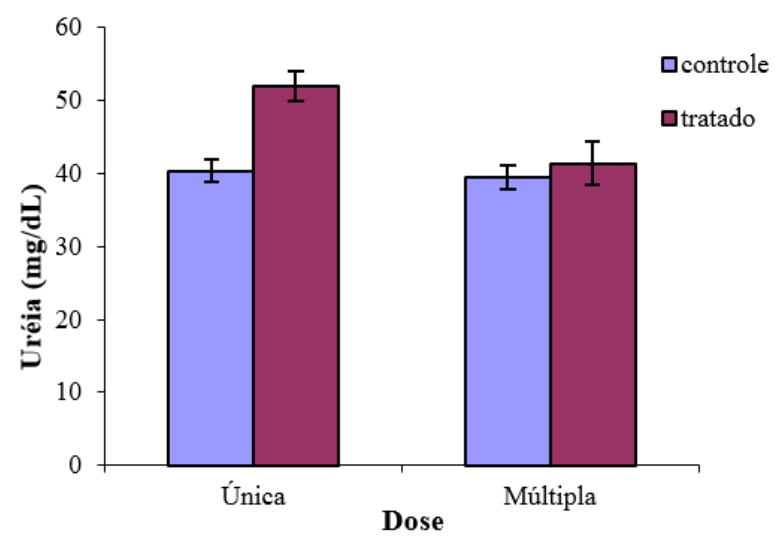

Fonte: Dados da pesquisa.

Esse resultado está em concordância com $\mathrm{Melo}^{10}$, que estudando um caso de nefrite intersticial aguda em criança também encontra uma dose aumentada de ureia, em relação aos valores de referência.

Em relação a creatinina (Figura 2) não se encontrou diferença estatística significativa em ambas as coletas, dose única $(\mathrm{p}>0,8)$ e doses múltiplas $(\mathrm{p}>0,08)$ durante quatro dias, isso justifica-se pelo fato desse curto tempo de administração da droga, que segundo Chahade ${ }^{14}$, o aumento da creatinina plasmática é visto durante os três a sete primeiros dias da terapia, tempo necessário para alcançar níveis estáveis da droga e máxima inibição da síntese de prostaglandinas.

Figura 2: Dosagem de creatinina nos dois tipos de tratamentos

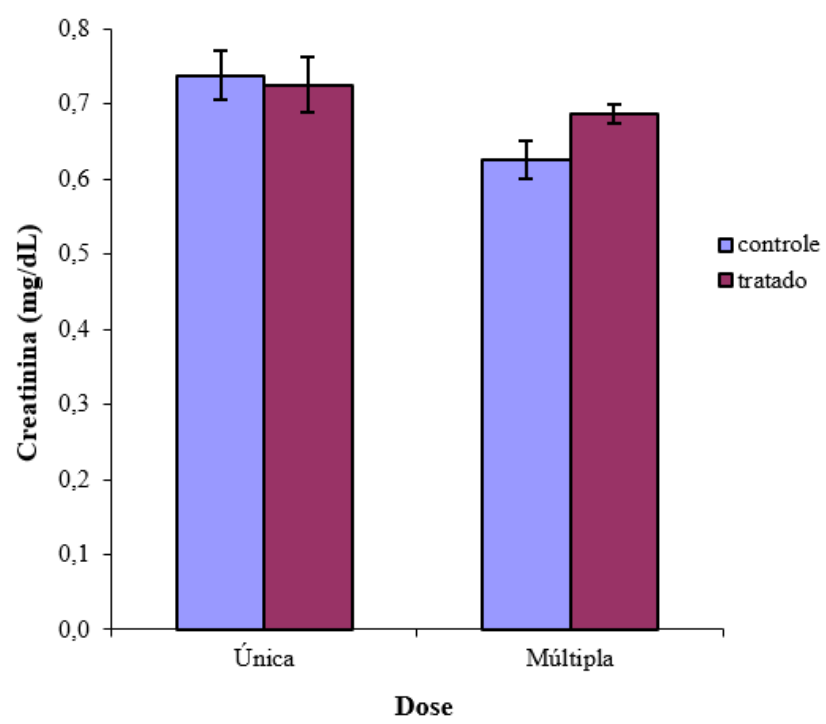

Fonte: Dados da pesquisa.

De acordo com Bricks ${ }^{11}$ além dos efeitos agudos sobre a função renal, os analgésicos podem causar doença renal crônica (necrose papilar e nefrite intersticial crônica), quando usados em doses excessivas.

A toxicidade renal pelo uso de AINEs tem sido muito estudada nos últimos anos. A possibilidade da dipirona induzir uma insuficiência renal aguda é um problema que preocupa a área de saúde, sobretudo a classe médica, por ser um fármaco amplamente consumido pela população.

\subsection{Resultados histopatológicos}

Segundo Sabatini ${ }^{12}$, além dos efeitos agudos sobre a função renal, os analgésicos podem causar doença renal crônica (necrose papilar e nefrite intersticial crônica), quando usados em doses excessivas; em doses habituais, não causa nefrotoxicidade; entretanto, em animais, observa-se lesão renal aguda após a ingestão de altas doses (500 a 1.000 mg/ $\mathrm{kg})$.

Os resultados histopatológicos apresentaram no rim do animal controle dose única (Figura 3A e 3B) que o parênquima do córtex renal apresenta aspectos normais, glomérulos renais e estruturas tubulares apresentando núcleos com cromatina finamente granular e homogênea (seta curta em Figura 3B1).

Figura 1: Fotomicrografias dos aspectos histopatológicos de rim de rato submetidos a dose única de dipirona (Coloração: Hematoxilina e Eosina. Aumento Final: 40X (em A e C), 200X em (B e D) e 400X em (B1 e D1)

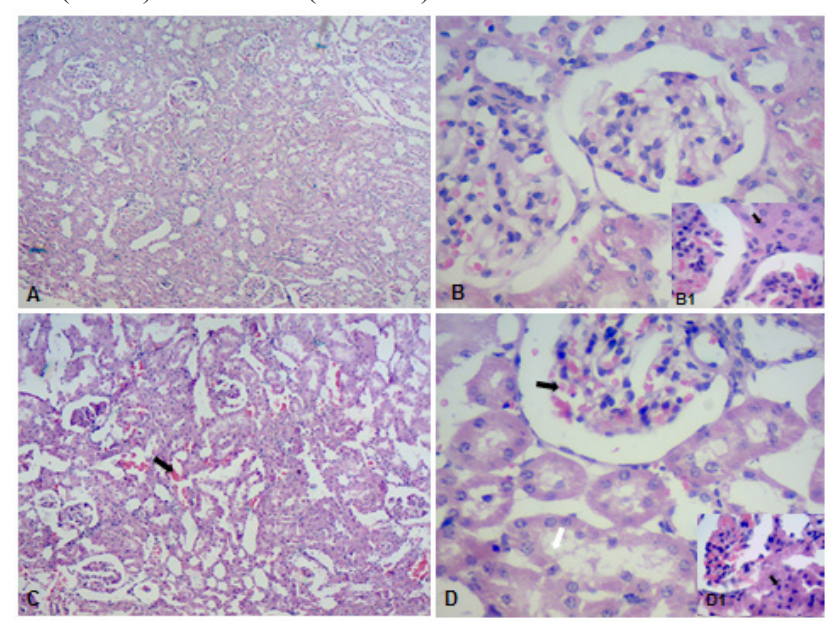

Fonte: Dados da pesquisa.

Entretanto, com o uso da dipirona dose única no grupo experimental (Figura 3C e 3D), observou-se o parênquima do córtex renal apresentando áreas hemorrágicas e inflamatórias com presença de linfócitos (seta preta), túbulos renais apresentando contornos celulares irregulares (seta branca) e núcleos picnóticos (seta curta em D1).

Encontrou-se diferenças significativas e de todos os parâmetros, somente na hemorragia glomerular na dose múltipla que a diferença não foi significativa, esses resultados se coadunam com Haase ${ }^{14}$ que em estudo de caso, o paciente com dose excessiva de dipirona apresentou quadro de nefrite intersticial 24 horas após a internação, detectado por meio de oligúria inicialmente e posteriormente uma anúria.

Observou-se na análise histopatológica do rim do animal controle dose múltipla (Figura 4A e 4B), o parênquima 
do córtex renal de aspectos normais, glomérulos renais e estruturas tubulares apresentando núcleos com cromatina finamente granular e homogênea.

Figura 4: Fotomicrografias dos aspectos histopatológicos de rim de rato submetido a doses múltiplas de dipirona (Coloração: Hematoxilina e Eosina. Aumento Final: 40X (em A e C), 200X (em B e D) e 400X (em B1 e D1)

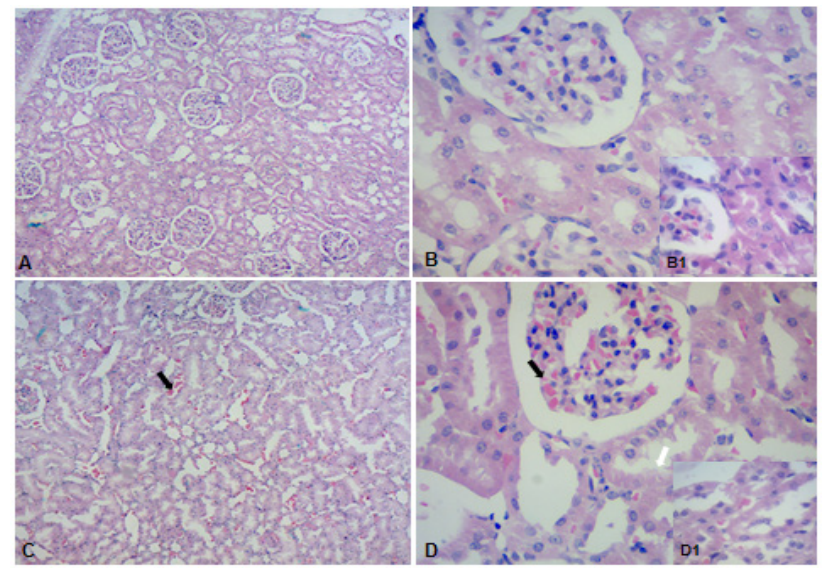

Fonte: Dados da pesquisa.

A histopatologia mostrou também a presença de infiltrado inflamatório intersticial com linfócitos em ambas as doses com diferença estatística significativa, sugerindo uma nefrite intersticial aguda NIA induzida por AINEs ${ }^{10}$.

Com o uso da dipirona dose múltipla, no grupo experimental (Figura 4C e 4D) observou-se que o parênquima do córtex renal apresentou áreas hemorrágicas em estruturas tubulares (seta preta em 4C) e glomerulares (seta curta).

Os três tipos de lesões no rim analisados no gráfico da Figura 5 (hemorragia glomerular $(p<0,008)$, congestão renal $(\mathrm{p}<0,02)$ e processo inflamatório $(\mathrm{p}<0,01)$, apresentaram médias significativamente diferentes nos grupos tratado com dose única e controle

Figura 6: Escores das lesões renais em dose única

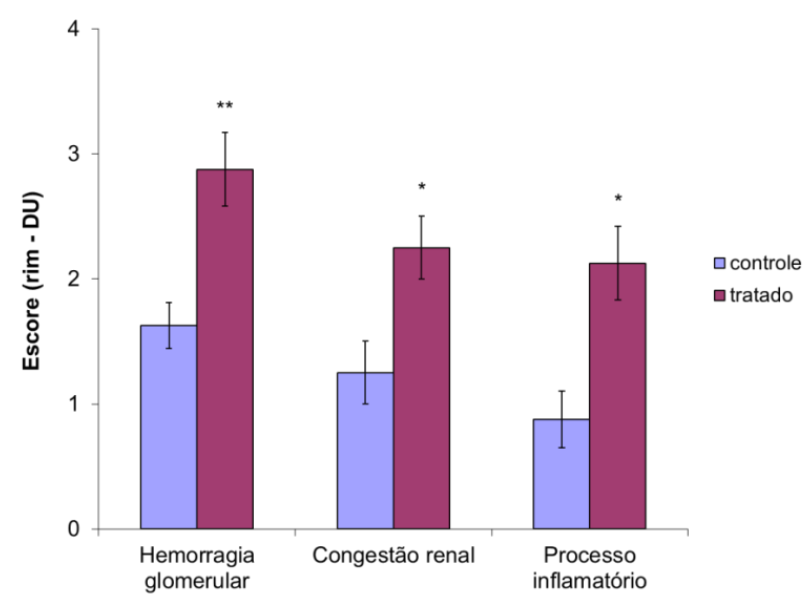

Fonte: Dados da pesquisa.

Os três tipos de lesões no rim analisados (4), hemorragia glomerular $(p<0,1)$, congestão renal $(p<0,01)$ e processo inflamatório $(\mathrm{p}<0,005)$ apresentaram médias significativamente diferentes nos grupos tratado com dose múltipla e controle, somente um parâmetro analisado, a hemorragia glomerular, não apresentou média significativa diferente.

Figura 7: Escores das lesões renais em doses múltiplas

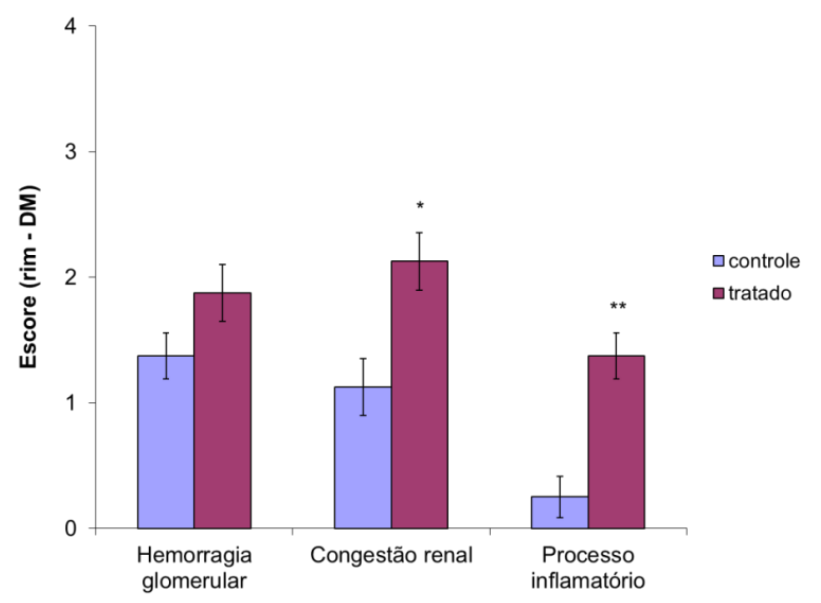

Fonte: Dados da pesquisa.

Para Melo et al..$^{10}$, o paracetamol, os AINEs e os antibióticos são os fármacos mais frequentemente prescritos na pediatria, e o seu uso tem aumentado exponencialmente ao longo das últimas décadas, muitas vezes sem prescrição médica. Os autores consideram fundamental refletir sobre o racionamento destes fármacos na idade pediátrica.

Esses resultados levam o profissional da área de saúde a uma atenção especial quanto ao uso indiscriminado de anti-inflamatórios não esteroidais (AINE, levando a uma necessidade de orientar e conscientizar a população quanto ao uso irresponsável da automedicação e suas consequências.

\section{Conclusão}

Os resultados do modelo experimental atestam que metodologia aplicada foi capaz de demonstrar as alterações histopatológicas do grupo tratado e a preservação das características normais do grupo controle, segundo o objetivo esperado. Consequentemente, as doses únicas e múltiplas aplicadas nos animais nos períodos estipulados, causou:

- Alterações histopatológicas no rim como: hemorragia glomerular, congestão renal e processo inflamatório;

- Alterações no marcador renal, ureia com diferença estatística significativa na dose única.

Os resultados obtidos comprovam a necessidade de um maior número de pesquisas que envolvam os aspectos morfológicos dos órgãos mais suscetíveis à ação da dipirona como fígado, sistema nervoso e cardiovascular, bem como discussões que envolvam políticas de saúde pública, que também incluem com propriedade o profissional de enfermagem. 


\section{Referências}

1. AME. Dicionário de Administração de Medicamentos na Enfermagem 2009/2010. Rio de Janeiro: EPUB; 2009.

2. Knappmann AL, Melo EB. Qualidade de medicamentos isentos de prescrição: um estudo com marcas de dipirona comercializadas em uma drogaria de Cascavel (PR, Brasil). Ciênc Saúde Coletiva 2010;15(3):3467-76.

3. Alves TA, Malafaia G. Self-medication among students of a higher education institution in Goiás. ABCS Health Sci 2014; 39(3):153-9

4. Andrade A. Uso de dipirona na automedicação. Blumenau: Universidade Federal de Blumenau; 2010.

5. Herdeg C, Hilt F, Buchtemann A, Bianchi L, Klein R. Allergic cholestatic hepatitis and exanthema induced by metamizole: verification by lymphocyte transformation test. Liver 2002;22:507-13.

6. Larini L. Fármacos e medicamentos. Porto Alegre: Artmed; 2008.

7. Melgaço SSC, Saraiva MIR, Lima TTC, Silva Júnior GB, Daher EF. Nefrotoxicidade dos anti-inflamatórios não esteroidais. Medicina (Ribeirão Preto) 2010;43(4):382-90.

8. Potter PA, Perry AG. Fundamentos de enfermagem: conceitos, processo e prática. Rio de Janeiro: Elsevier; 2005.
9. Peres LAB, Delfino VDA, Tutida LA, Faris BA, Barbosa DS, Favero ME, et al. Efeitos do Ramipril, Captopril e Sinvastatina na injúria da preservação a frio de rins de ratos. J Bras Nefrol 2009;31(1):39-47.

10. Melo C, Silva RS, Tomé S, Carvalho S, Teixeira P. Nefrite intersticial induzida por fármacos. Acta Pediatr Port 2013;44(3):130-3.

11. Bricks LF. Analgesics, antipyretics and antiinflammatory drugs: toxicity - 1st part Pediatria (São Paulo) 1998;20(2):12736.

12. Sabatini S. Pathophysiologic mechanism in analgesicinduced papillary necrosis. Am J Kidney Dis 1996;28:S34-8.

13. Haase D, Hübner S, Kunellism S, Kotzerke G, König H. Metamizol suicide - lethal outcome despite maximum therapy. Toxichem Krimtech 2012;79(2):71-9.

14. Chahade WH, Giorgi RDN, Szajubok JCM. Anti-inflamatórios não hormonais. Einstein (São Paulo) 2008;6:S166-74.

15. Zanuzzo FS. Efeitos da dipirona, do meloxicam e da associação dipirona e meloxicam sobre a hemostasia em cães conscientes e sobre o controle da dor pós-operatória em cadelas submetidas à ovariosalpingohisterectomia, Dissertação [Mestrado em Medina] - Universidade Estadual Paulista; 2014. 